\title{
Effect of Demographic Variables on Organizational Role Stress and Burnout: an Empirical Investigation
}

\author{
Subhash R Soni ${ }^{1}$, Vyas J M ${ }^{1}$, Pestonjee D M², Kher H N ${ }^{3}$, Thakkar K A ${ }^{3}$ and Vijaya Lakshmi $\mathrm{Y}^{4}$ \\ ${ }^{1}$ Gujarat Forensic Sciences University, Gandhinagar, Gujarat, India \\ ${ }^{2}$ School of Petroleum Management, Pandit Deen Dayal Petroleum University, Gandhinagar, Gujarat, India \\ ${ }^{3}$ Sardarkrushinagar Dantiwada Agricultural University, Sardarkrushinagar, Gujarat, India \\ ${ }^{4}$ District Institute of Education and Training, Gandhinagar, Gujarat, India
}

*Corresponding author: Subhash R Soni, Research Scholar, Gujarat Forensic Sciences University, Gandhinagar, Gujarat, India, Tel: 91 9978407023; Fax: 9179 23254603; E-mail: madhuspl@yahoo.com

Received Date: December 06, 2014, Accepted Date: December 26, 2014, Published Date: January 02,2015

Copyright: () 2015, Subhash R Soni, et al. This is an open-access article distributed under the terms of the Creative Commons Attribution License, which permits unrestricted use, distribution, and reproduction in any medium, provided the original author and source are credited.

\begin{abstract}
Objective: To examine the relationship of a set of independent variables (age, qualification, experience, position, income and marital status) with occupational stress (Organizational Role Stress (ORS) \& Burnout) among Animal Husbandry Personnel.
\end{abstract}

Setting: Eight districts of Gujarat state, India

Design: Descriptive cross-sectional

Subjects: Two hundred and thirty six (236) Veterinarians and One hundred and forty six (146) Para Veterinarians of Animal Husbandry Department

Outcome measures: The findings relate to the status of Burnout and ORS factors in case of Veterinarians and Para Veterinarians of Animal Husbandry Department. The statistical treatment established the various possibilities of 'cause-effect' relationship by applying Multiple Regression Analysis and Path analysis.

Methods: The Pareek's Occupational Stress scale and Maslach Burnout scale (MBI-GS) were used for data collection, while central tendencies, frequency, t-test, Co-efficient of Correlation (' $r$ '), Multiple regression analysis, Standard Partial Regression Co-efficient, Stepwise multiple regression analysis and Path co-efficient analysis were used for statistical analysis.

Results: The data revealed that maximum respondents had education of graduation level with above 21 years service experience (62.0\%) with monthly income in the range of Rs.20,001 to Rs.50,000 (69.6\%). Majority of them were from age group of 36 to 50 years $(59.5 \%)$. Maximum number of veterinarians and paraveterinarians had high IRD, RS, REC, RO, PI and RA. On the other hand, maximum respondents in case of RE were under low category. Considering Role Isolation, nearly $40 \%$ of veterinarians $(39.83 \%)$ and paraveterinarians $(38.36 \%)$ were under high category. Almost equal distribution of $33 \%$ each in low, medium and high categories of SRD was observed, in case of veterinarians. Length of service and age had negatively significant relationship with RS and PI, Age had negatively significant relationship with RIN. Similarly, length of service had negatively significant relationship with REC. Educational qualification had positively significant relationship with RE and monthly income had negatively significant relationship with $\mathrm{PI}$. The data revealed that majority of veterinarians and paraveterinarians were having low level of Exhaustion and Cynicism. The Personal Efficacy was found to be high in both the categories of the employees. All the six demographic variables failed to establish significant relationship with three sub-scales of burnout viz., Exhaustion, Cynicism and Personal Efficacy.

Conclusion: The results lead us to conclude that there is a need for more proactive HR policies to maintain and alleviate the role stress and burnout. It can be recommended that the organization needs to mandate a 'stress audit' for various categories of personnel. Counseling may also be provided on a case to case basis so that individuals may be helped with their cognitive restructuring.

Keywords: Veterinarians; Paraveterinarians; Organizational Role Stress; Step-wise multiple regression; Path analysis; Burnout 


\section{Introduction}

Stress becomes a universfeature of and no one can live without experiencing some degree of stress. As a result, stress related diseases and syndromes are becoming very common today. The pressure of balancing the work life and non work life is also at peak. This also often results in increasing stress and burnout among the employees. Stress has been considered as one of the major factors in work organization. Sources of stressors in the employment organization are work, role, personal development, interpersonal relations and organization climate. The animal husbandry sector which is potentially one of the most important sectors of rapid socioeconomic development of Gujarat State, India, is also not exceptional to this $[1,2]$.

The veterinary and animal science service is a highly specialized area that involves tasks like management and health care of the livestock and poultry, prevention of diseases, disease diagnosis etc. Also, Globalization and implementation of world trade agreements (WTA) has increased the scope for export of livestock and hygienically produced livestock products manifolds. Movement of livestock and livestock products across the borders also introduces the risk of transboundary infections. Zoonoticdiseases and public health issues associated with livestock and livestock products are other concerns.

In this context, the role of the veterinarian and paraveterinarian of Animal husbandry Department has become multifaceted as a clinician, researcher, and an advisor. In order to manage these multi dimensional activities, there is a high need of qualified technical manpower and facilities. However, Shortage of technical manpower and poor Veterinary infrastructure is affecting quality of manpower and services. Only 34,500 veterinarians are employed for field services against the requirement of 67,000 . Similarly, against the requirement of 7500 veterinary scientists for teaching and research, only 3050 are available. Availability of Para-vets and other supporting staff is only 52,000 against the requirement of 2,59,000 (Source: 12th five year plan (2012-17) of Gujarat Government).The main duties of these cadres include organizing animal health camps at village level; provide animal health care service at the door step; preventive as well as curative service delivery such as vaccination and educating animal keepers. The factors contributing to burnout syndrome and ORS among veterinarians and paraveterinarians include boredom, physical working conditions, time pressure and deadlines, work demands, critical decision making in emergency situations, over expectations of people, job design and technical problems.

Under such conditions, the employees of animal husbandry are undervalued and under-appreciated in the society. The veterinarians and paraveterinarians are confronted with heart-breaking and frightening conditions that make them angry, frustrated, and hopeless. We observed the following symptoms among these employees: loss of interest in service, aggressive behavior, aloofness, absenteeism, lack of satisfaction for stake holders, decreasing productivity and production, lack of dedication and timely appropriate response, increasing complaints about professional service delivery and avoidance of responsibility.

There is no research work available on burnout syndrome as well as organizational role stress (ORS) among these employees. It was deemed fit to investigate the ORS and burnout level among these cadres as certain problems and issues can be resolved at management level. A detailed discussion was held with the regional Joint Director, Animal Husbandry department and mutual agreement arrived at with him. It was decided to undertake stress audit of these employees of the department with ORS scale and MBG-GS inventory along with certain informative questionnaire about demographic variables.

The relationship between demographic variables viz., age, qualification, experience, position, income and marital status and stress reviewed through available literature. There are inconsistent evidences in the literature as regards to the influence of age on organizational role stress and burnout. Age was found to be negatively associated with role stress, but was positively related to organizational effectiveness [3], and also was a predictor of burnout behavior [4]. A decline in the level of burnout was reported as the age increased [5-7]. Price and Spence on the basis of their study on burnout among drug and alcohol counseling employees provided empirical evidence that higher level of burnout was experienced by younger employees. The study specifically mentioned elevated range of score on emotional exhaustion and depersonalization dimensions of burnout among younger employees. Qualification acts as mediator, either increases or reduces stress depending on perspective of the individuals. Ansari revealed that the correlation between the nature of stress and qualification of teachers in different cadres was found to be nonsignificant [8]. The length of service has negative and positive relationship with stress. Even then more studies revealed that individual with lesser experience, experienced more stress as compared to the individual with more service years [9-11]. The position of the worker increases, the stress level also increases [12,13]. Kumar found that unmarried executives experienced significantly higher stress as compared to married executives. Executives married to working women were observed to experience significantly high total role stress in comparison to executives married to housewives [14].

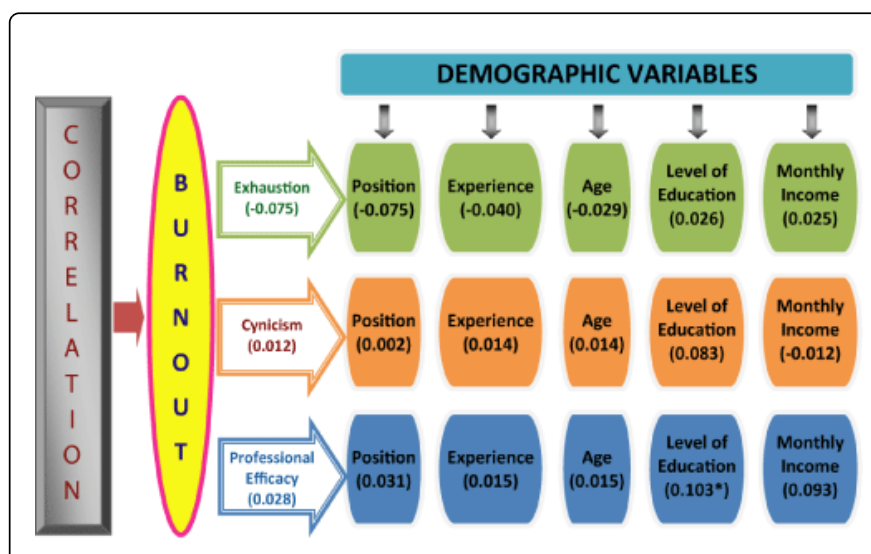

Figure 1: Correlation between Demographic Variables and Burnout Subscales

With this background in mind, the present study was taken-up to understand the effect of certain demographic factors on the organizational role stress and burnout among veterinarians and paraveterinarians of Gujarat State.

The following are the research questions on which the study is based upon:

What are the demographic characteristics of animal husbandry personnel?

What is the level of occupational stress in animal husbandry personnel? 
Do the demographic factors (age, qualification, experience, position, income and marital status) explain the differences in occupational stress level of animal husbandry personnel?

\section{Data and Method}

\section{Sample of the study}

A total of 236 veterinarians and 146 paraveterinarians from eight districts of Gujarat state who are working in Animal Husbandry Department were selected randomly and these people constitute the sample of the study.

\section{Tools for data collection}

\section{Organizational Role Stress scale (ORS Scale)}

Organizational Role Stress Scale (ORS scale) a standardized scale developed by Pareek was used to assess the level of role stress among the veterinarians and paraveterinarians. The respondents based on the frequencies of their response for each role stress were classified into three categories viz., high, medium and low [15].

\section{Burnout sub-scale}

To study the burnout phenomenon, the MBI - General Survey or MBI-GS developed by [16] was used. The MBI-GS provides a three dimensional perspective on burnout. A high degree of burnout is reflected in high scores on Exhaustion and Cynicism and low scores on professional efficacy. The respondents based on three sub-scales of burnout were classified into three categories viz., high, medium and low.

\section{Data collection}

With the consent from the Joint Director of Animal Husbandry Department, Gujarat State Government, the data on demographic variables, ORS scale and MBI-GS inventory was collected through administering questionnaire from respondents. Thus, the data was collected from 382 personnel (236 Veterinarians and 146 Para Veterinarians) of animal husbandry department. The employees were assured of confidentiality of the data.

\section{Statistical frame work for analysis of the data}

The data was analyzed using the Statistical Package for Social Sciences (SPSS). The following statistical methods were used in the present study.

a. Frequency distribution, simple averages and percentage was used to analyze the data. Mean score was calculated for finding out different values and Standard Deviation was used for classification of the respondents into different categories.

b. Where ever required, the Pearson's product moment correlation co-efficient was computed to find out the relationship between each of the independent variables and dependent variables.

c. To ascertain the effect of different selected characteristics on the extent of organizational role stress and burnout of the respondents, regression analysis was employed. In order to select the independent variables to be included in the multiple regression equation, the correlation between different characteristics and the organizational role stress and burnout was examined. Variables having significant and high correlation with organizational role stress and burnout were used as independent variables to the multiple regressions model. Where ever required, the partial regression coefficients were tested for their significance.

d. Standard Partial Regression Co-efficient (SPRC) was used to free the obtained values from units of measurement and facilitate comparison among them.

e. Step-wise multiple regression analysis was carried out to determine the important variables with their predictive ability in explaining the variation in the dependent variable. The analysis was continued until the last variant of which additional contribution was the least.

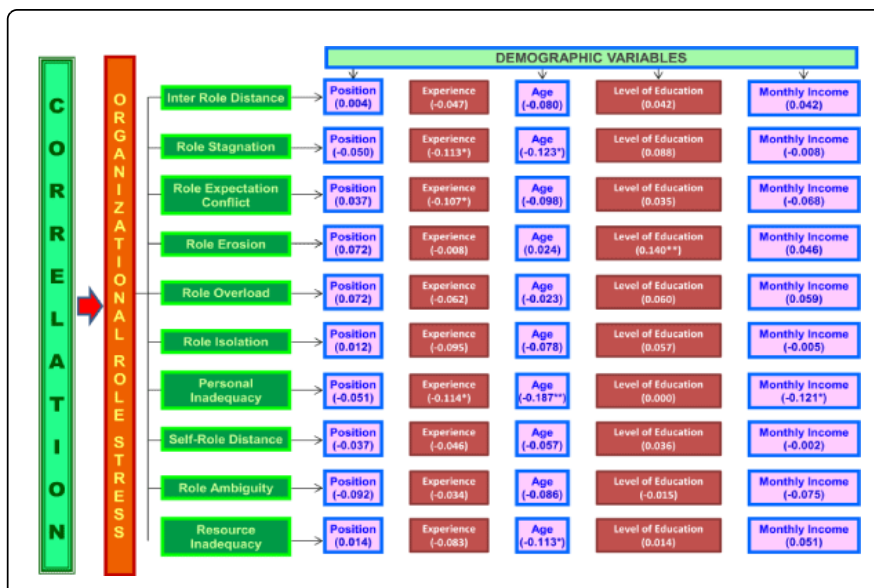

Figure 2: Correlation between Demographic Variables and Organizational Role Stress factors

f. Path analysis adopting multivariable path model as suggested by Dewey and $\mathrm{Lu}$ [17] was used to isolate the direct, indirect and substantial effect of independent variables on dependent variable i.e., organizational role stress and burnout.

\section{Results and Discussion}

\section{Demographic characteristics}

Inventories were selected on the basis of the objectives of the study. Since the respondents were highly educated, questionnaire was considered to be the most suitable instrument for the data collection. The demographic characteristics schedule consisted information of the animal husbandry personnel regarding age, qualification, experience, position, income and marital status. The data regarding demographic characteristics is presented in Table 1. It can be inferred from this table that nearly two-fifth of the respondents $(60.7 \%)$ had education of graduation level, having above 21 years of service experience $(62.0 \%)$ with monthly income of Rs.20,001 to Rs.50,000 (69.6\%). Majority of them were from age group of 36 to 50 years (59.5\%). Only two employees were unmarried. 
Citation: Soni SR, Vyass JM, Pestonjee DM, Kher HN, Thakkar KA, et al. (2015) Effect of Demographic Variables on Organizational Role Stress and Burnout: an Empirical Investigation. J Psychiatry 18: 233. doi:10.4172/Psychiatry.1000233

Page 4 of 12

\begin{tabular}{|c|c|c|c|c|c|}
\hline \multirow{3}{*}{$\begin{array}{l}\text { Sr. No. } \\
1 .\end{array}$} & \multirow{3}{*}{$\begin{array}{l}\text { Character } \\
\text { Position }\end{array}$} & \multicolumn{2}{|c|}{ Category } & \multirow{2}{*}{$\begin{array}{l}\text { Frequency } \\
236\end{array}$} & \multirow{2}{*}{\begin{tabular}{|l|} 
Per cent \\
61.8
\end{tabular}} \\
\hline & & (i) & Veterinarian & & \\
\hline & & (ii) & Paraveterinarian & 146 & 38.2 \\
\hline \multirow{3}{*}{2.} & \multirow{3}{*}{ Level of education } & (i) & Under-Graduate & 122 & 31.9 \\
\hline & & (ii) & Graduate & 232 & 60.7 \\
\hline & & (iii) & Post-Graduate and Above & 28 & 7.3 \\
\hline \multirow{7}{*}{3.} & \multirow{7}{*}{ Length of service (Experience) years } & (i) & 0 to 5 & 44 & 11.5 \\
\hline & & (ii) & 6 to 10 & 23 & 06.0 \\
\hline & & (iii) & 11 to 15 & 65 & 17.0 \\
\hline & & (iv) & 16 to 20 & 13 & 03.4 \\
\hline & & (v) & 21 to 25 & 118 & 30.9 \\
\hline & & (vi) & 26 to 30 & 52 & 13.6 \\
\hline & & (vii) & Above 30 & 67 & 17.5 \\
\hline \multirow{4}{*}{4.} & \multirow{4}{*}{ Monthly income (Rs/-) } & (i) & Up to 10,000 & 32 & 08.4 \\
\hline & & (ii) & $10001-20000$ & 27 & 07.1 \\
\hline & & (iii) & $20001-50,000$ & 266 & 69.6 \\
\hline & & (iv) & Above 50,000 & 57 & 14.9 \\
\hline \multirow{9}{*}{5.} & \multirow{9}{*}{ Age (in years) } & (i) & 21 to 25 & 02 & 00.5 \\
\hline & & (ii) & 26 to 30 & 40 & 10.5 \\
\hline & & (iii) & 31 to 35 & 29 & 07.6 \\
\hline & & (iv) & 36 to 40 & 58 & 15.2 \\
\hline & & (v) & 41 to 45 & 87 & 22.8 \\
\hline & & (vi) & 46 to 50 & 82 & 21.5 \\
\hline & & (vii) & 51 to 55 & 51 & 13.4 \\
\hline & & (viii) & 56 to 60 & 31 & 08.1 \\
\hline & & (ix) & Above 60 & 02 & 00.5 \\
\hline \multirow{2}{*}{6.} & \multirow{2}{*}{ Marital status } & (i) & Married & 380 & 99.5 \\
\hline & & (ii) & Unmarried & 002 & 00.5 \\
\hline
\end{tabular}

Table 1: Distribution of the respondents according to their demographic characteristics ( $n=382$ )

\section{Organizational role stress}

The data regarding organizational role stress among the respondents is given in Table 2 . This table indicates that maximum of veterinarians (72.46\%) and paraveterinarians (77.40\%) had high IRD. In case of RS also, maximum of veterinarians (57.63\%) and paraveterinarians $(47.26 \%)$ were observed having high RS. The similar trend was observed in case of REC as maximum of the respondents of both the categories were in high category of REC. Looking to RE, maximum of veterinarians (54.66\%) and paraveterinarians $(51.37 \%)$ were in low category. In case of RO, $55.51 \%$ of veterinarians and $60.96 \%$ of paraveterinarians were under high category of role stress.
Considering Role Isolation, nearly $40 \%$ of veterinarians and paraveterinarians were under high category.

Nearly, half of the veterinarians $(50.42 \%)$ and paraveterinarians (47.26\%) were under high category of PI. Almost equal distribution of $33 \%$ each in low, medium and high categories of SRD was observed in two categories of the respondents. In case of RA, a few veterinarians $(24.58 \%)$ and paraveterinarians $(31.51 \%)$ were under low category. Looking to RIN, maximum veterinarians (61.02 \%) and paraveterinarians $(63.70 \%)$ were under high category. 
Citation: Soni SR, Vyass JM, Pestonjee DM, Kher HN, Thakkar KA, et al. (2015) Effect of Demographic Variables on Organizational

Page 5 of 12

\section{Burnout}

The data regarding burnout among the respondents is given in Table 3. This table indicates that in case of Exhaustion sub-scale, the higher percentage of both veterinarians (49.15\%) and paraveterinarians $(54.79 \%)$ were in low category. In case of Cynicism, maximum number of both veterinarians $(50 \%)$ and paraveterinarians (50.68\%) were in medium category. Looking to professional Efficacy, nearly half of veterinarians (46.19\%) and paraveterinarians $(47.95 \%)$ were found having high PE.

\begin{tabular}{|c|c|c|c|c|c|c|}
\hline \multirow{2}{*}{$\begin{array}{l}\text { Sr. } \\
\text { No. }\end{array}$} & \multirow{2}{*}{ Name of ORS } & \multirow{2}{*}{ Category } & \multicolumn{2}{|c|}{ Veterinary officers } & \multicolumn{2}{|c|}{ Paraveterinarians } \\
\hline & & & Frequency & Percent (\%) & Frequency & Percent (\%) \\
\hline \multirow{3}{*}{1.} & \multirow{3}{*}{ Inter Role Distance } & Low & 28 & 11.86 & 10 & 6.85 \\
\hline & & Medium & 37 & 15.68 & 23 & 15.75 \\
\hline & & High & 171 & 72.46 & 113 & 77.40 \\
\hline \multirow{3}{*}{2.} & \multirow{3}{*}{ Role Stagnation } & Low & 59 & 25.00 & 38 & 26.03 \\
\hline & & Medium & 41 & 17.37 & 39 & 26.71 \\
\hline & & High & 136 & 57.63 & 69 & 47.26 \\
\hline \multirow{3}{*}{3.} & \multirow{3}{*}{ Role Expectation Conflict } & Low & 67 & 28.39 & 36 & 24.66 \\
\hline & & Medium & 66 & 27.97 & 44 & 30.14 \\
\hline & & High & 103 & 43.64 & 66 & 45.21 \\
\hline \multirow{3}{*}{4.} & \multirow{3}{*}{ Role Erosion } & Low & 129 & 54.66 & 75 & 51.37 \\
\hline & & Medium & 57 & 24.15 & 39 & 26.71 \\
\hline & & High & 50 & 21.19 & 32 & 21.92 \\
\hline \multirow{3}{*}{5.} & \multirow{3}{*}{ Role Overload } & Low & 37 & 15.68 & 21 & 14.38 \\
\hline & & Medium & 68 & 28.81 & 36 & 24.66 \\
\hline & & High & 131 & 55.51 & 89 & 60.96 \\
\hline \multirow{3}{*}{6.} & \multirow{3}{*}{ Role Isolation } & Low & 85 & 36.02 & 49 & 33.56 \\
\hline & & Medium & 57 & 24.15 & 41 & 28.08 \\
\hline & & High & 94 & 39.83 & 56 & 38.36 \\
\hline \multirow{3}{*}{7.} & \multirow{3}{*}{ Personal Inadequacy } & Low & 41 & 17.37 & 22 & 15.07 \\
\hline & & Medium & 76 & 32.20 & 55 & 37.67 \\
\hline & & High & 119 & 50.42 & 69 & 47.26 \\
\hline \multirow{3}{*}{8.} & \multirow{3}{*}{ Self-Role Distance } & Low & 79 & 33.47 & 56 & 38.36 \\
\hline & & Medium & 79 & 33.47 & 48 & 32.88 \\
\hline & & High & 78 & 33.06 & 42 & 28.77 \\
\hline \multirow{3}{*}{9.} & \multirow{3}{*}{ Role Ambiguity } & Low & 58 & 24.58 & 46 & 31.51 \\
\hline & & Medium & 84 & 35.59 & 48 & 32.88 \\
\hline & & High & 94 & 39.83 & 52 & 35.62 \\
\hline \multirow{3}{*}{10.} & \multirow{3}{*}{ Resource Inadequacy } & Low & 48 & 20.34 & 25 & 17.12 \\
\hline & & Medium & 44 & 18.64 & 28 & 19.18 \\
\hline & & High & 144 & 61.02 & 93 & 63.70 \\
\hline
\end{tabular}

Table 2: Categorization of respondents according to ORS scale $(n=382)$ 
Citation: Soni SR, Vyass JM, Pestonjee DM, Kher HN, Thakkar KA, et al. 2015) Effect of Demographic Variables on Organizational Role Stress and Burnout: an Empirical Investigation. J Psychiatry 18: 233. doi:10.4172/Psychiatry.1000233

Page 6 of 12

\begin{tabular}{|c|c|c|c|c|c|c|}
\hline \multirow{2}{*}{ Sr. No. } & \multirow{2}{*}{ Burnout sub-scales } & \multirow{2}{*}{ Category } & \multicolumn{2}{|c|}{ Veterinary officers } & \multicolumn{2}{|c|}{ Paraveterinarian } \\
\hline & & & Frequency & Percent (\%) & Frequency & Percent (\%) \\
\hline \multirow{3}{*}{1.} & \multirow{3}{*}{ Exhaustion } & Low & 116 & 49.15 & 80 & 54.79 \\
\hline & & Medium & 92 & 38.98 & 49 & 33.56 \\
\hline & & High & 28 & 11.86 & 17 & 11.64 \\
\hline \multirow{3}{*}{2.} & \multirow{3}{*}{ Cynicism } & Low & 83 & 35.17 & 51 & 34.93 \\
\hline & & Medium & 118 & 50.00 & 74 & 50.68 \\
\hline & & High & 35 & 14.83 & 21 & 14.38 \\
\hline \multirow{3}{*}{3.} & \multirow{3}{*}{$\begin{array}{l}\text { Personal } \\
\text { Efficacy }\end{array}$} & Low & 83 & 35.17 & 41 & 28.08 \\
\hline & & Medium & 44 & 18.64 & 35 & 23.97 \\
\hline & & High & 109 & 46.19 & 70 & 47.95 \\
\hline
\end{tabular}

Table 3: Categorization of respondents according to burnout

\section{Relationship between demographic variables and organizational role stress}

Of the six demographic variables, marital status was not considered to examine its relationship with ORSs as only two respondents were found unmarried. The data in this regard is presented in Table 4 and shown as Figure 1. Job stress differs from person to person; such differences may be due to certain characteristics of the person. An attempt was, therefore, made to know the relationship of selected demographic variables with each of the ORS. It is evident from the data that all the demographic variables viz., position, length of service (experience), age, level of education and monthly income have failed to establish significant relationship with IRD, RI, SRD, RA, RIN and $\mathrm{RO}$ as the value of correlation coefficients were non-significant in all the cases. On the other hand, length of service (Experience) established negative significant relationship with RS (-0.113), REC (-0.107) and PI $(-0.114)$ at 0.05 level of significance. This indicates that employee with more experience generally have low RS, REC and PI.

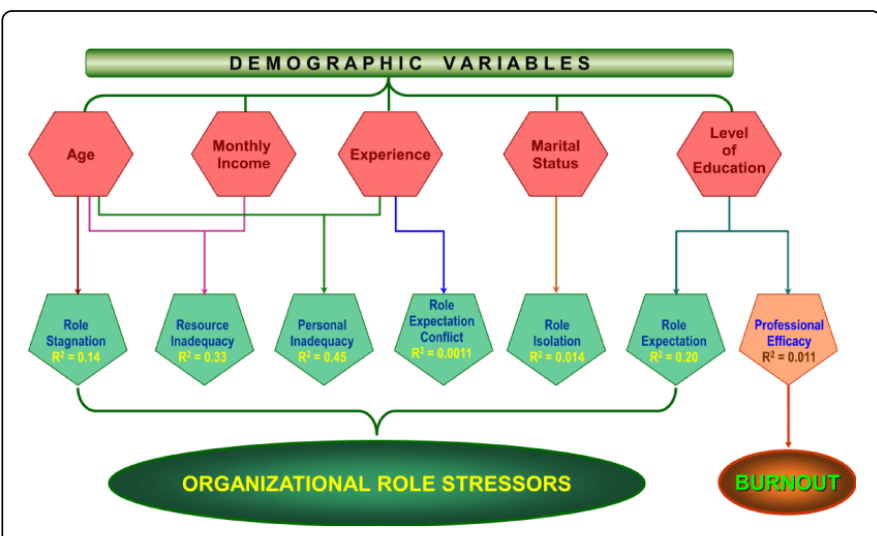

Figure 3: Regression of Demographic Variables and Organizational Role Stress factors and Burnout
Age also established negative significant relationship with RS $(-0.123)$ and RIN (-0113) as ' $r$ ' values are negatively significant at 0.05 level and with PI (-0.187) at 0.01 level. Level of education established positively significant relationship only with $\mathrm{RE}$ as the ' $r$ ' value of 0.140 is significant at 0.01 level of significance.

The variable, monthly income established negative significant relationship only with PI as ' $r$ ' value $(-0.121)$ was significant at 0.05 level of significance.

\section{Step-wise multiple regression of independent variables with organizational role stress factors}

Step-wise regression is widely adopted in multiple regression [18-22]. It has the added advantage that at each stage of analysis, every variable is subjected to an examination for its predictive value. Based on this approach, the stepwise multiple regression analysis was carried out to know the important variables with their predictive ability in explaining the variation in the dependent variable. In step-wise regression analysis, forward equation was used. In forward equation, the variables which were found having significant influence upon ORS were fitted. The data in this regard is presented in Table 5. Only age was found to contribute significantly to RS and hence, it was considered for stepwise multiple regressions. It is clear from table that age has explained $14 \%$ of total variation in respondents' $R S$. The ' $t$ ' value (-2.408) was found significant at 0.05 level of significance indicating its significant contribution in RS. The partial regression coefficient indicated that one unit change in age would change -0.313 units in respondents' RS. 
Citation: Soni SR, Vyass JM, Pestonjee DM, Kher HN, Thakkar KA, et al. (2015) Effect of Demographic Variables on Organizational Role Stress and Burnout: an Empirical Investigation. J Psychiatry 18: 233. doi:10.4172/Psychiatry.1000233

Page 7 of 12

\begin{tabular}{|c|c|c|c|c|c|}
\hline \multirow{2}{*}{ ORS } & \multicolumn{5}{|c|}{ Demographic variables } \\
\hline & Position & $\begin{array}{l}\text { Length of service (Experience) (In } \\
\text { years) }\end{array}$ & Age (In years) & Level of Education & Monthly Income (In Rs. \\
\hline IRD & 0.004 & -0.047 & -0.080 & 0.042 & 0.042 \\
\hline RS & -0.050 & $-0.113^{*}$ & $-0.123^{*}$ & 0.088 & -0.008 \\
\hline REC & 0.037 & $-0.107^{*}$ & -0.098 & 0.035 & -0.068 \\
\hline RE & 0.072 & -0.008 & 0.024 & $0.140^{* *}$ & 0.046 \\
\hline RI & 0.012 & -0.095 & -0.078 & 0.057 & -0.005 \\
\hline $\mathrm{PI}$ & -0.051 & $-0.114^{*}$ & $-0.187^{* *}$ & 0.000 & $-0.121^{*}$ \\
\hline SRD & -0.037 & -0.046 & -0.057 & 0.036 & -0.002 \\
\hline RA & -0.092 & -0.034 & -0.086 & -0.015 & -0.075 \\
\hline RIN & 0.014 & -0.083 & $-0.113^{*}$ & 0.014 & 0.051 \\
\hline RO & 0.072 & -0.062 & -0.023 & -0.060 & 0.059 \\
\hline
\end{tabular}

Table 4: Relationship between demographic variables and ORS factors

\begin{tabular}{|l|l|l|l|l|}
\hline $\begin{array}{l}\text { Sr. } \\
\text { No. }\end{array}$ & Demographic variable & $\begin{array}{l}\text { Partial regression co- } \\
\text { efficient (bi) }\end{array}$ & S.E. of bi 't' value \\
\hline RS & Age Group & -0.313 & 0.130 & $-2.408^{*}$ \\
\hline \multirow{2}{*}{ RIN } & Age & -0.5516 & 0.162 & $-3.428^{* *}$ \\
\cline { 2 - 5 } & Monthly income & 1.0640 & 0.381 & $2.795^{*}$ \\
\hline \multirow{2}{*}{ PI } & Age & -0.892 & 0251 & $-3.559^{* *}$ \\
\cline { 2 - 5 } & Experience & 0.453 & 0.228 & 1.982 \\
\hline RI & Marital status & 7.111 & 3.016 & $2.358^{*}$ \\
\hline RE & Experience & -0.230 & 0.110 & $-2 . .096^{*}$ \\
\hline
\end{tabular}

Table 5: Step-wise multiple regression of independent variable with ORS factors, $r 2=0.14$ (RS), 0.33 (RIN), 0.45 (PI), 0.14 (RI), 0.11 (REC), 0.20 $(\mathrm{RE}),{ }^{*}$ Significant at 0.05 level of significance, ${ }^{* *}$ Significant at 0.01 level of significance

Two variable viz., age and monthly income were found to contribute significantly to RIN and hence, both were considered for stepwise multiple regressions. It is clear from table that age + monthly income jointly explained $33 \%$ of total variation in respondents' RIN. The ' $t$ ' value in case of age was -3.428 and in case of monthly income was 2.795, which were found significant at 0.01 and 0.05 level of significance respectively indicating their significant contribution in RIN. The partial regression co-efficient indicated that one unit change in age and monthly income would change -0.5516 units and 1.0640 change in respondents' RIN, respectively. Out of five demographic variables two were found to contribute significantly to PI and hence, both were considered for stepwise multiple regressions. It is clear from the table that age + experience combined explained $45 \%$ of total variation in respondents' PI. The ' $t$ ' value in case of age was negatively significant (-3.559) and in case of experience, it was positively significant (1.982) indicating their significant contribution in PI. 


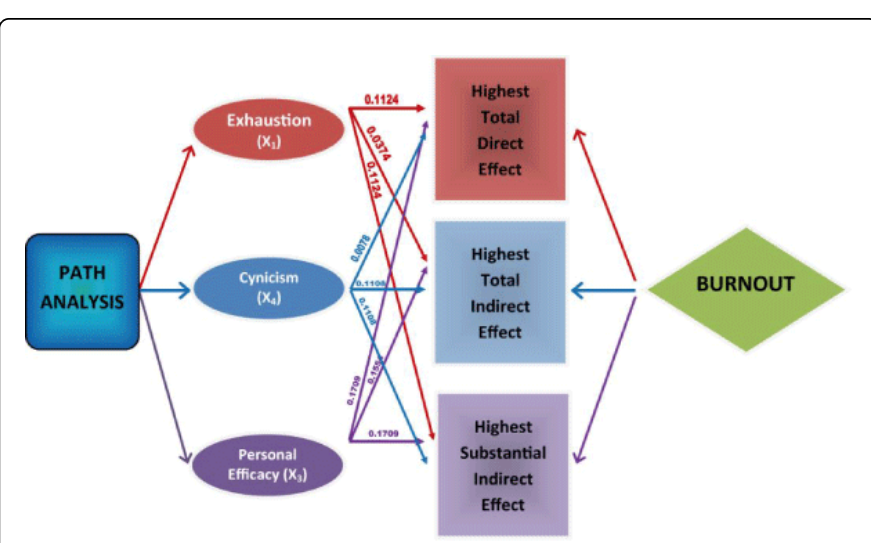

Figure 4: Path Analysis of Dependent Variables with Burnout Subscale

The partial regression co-efficient indicated that one unit change in age and monthly income would change -0.892 units and 0.453 units in respondents' PI, respectively. Marital status was found to contribute significantly to RI and hence, it was considered for stepwise multiple regressions. It is clear from the table that only marital status has explained $14 \%$ of total variation in respondents' RI. The't' value (2.358) was found significant at 0.05 level of significance indicating its significant contribution in RI. The partial regression co-efficient indicated that one unit change in age would change 7.111 units in respondents' RI. Only experience was found to contribute significantly to REC and hence, it was considered for stepwise multiple regressions. It is clear from table that only experience has explained $11 \%$ of total variation in respondents' REC. The ' $t$ ' value (-2.096) was found significant at 0.05 level of significance indicating its significant contribution in REC. The partial regression co-efficient indicated that one unit change in age would change - 0.230 units in respondents' REC. It could be seen from above table that level of education was found to contribute significantly to RE and hence it was considered for step-wise multiple regression. It is clear from the table that only level of education has explained $20 \%$ of total variation in respondents' RE. The ' $t$ ' value (2.757) was found significant at 0.05 level of significance indicating its significant contribution in RE. The partial regression coefficient indicated that one unit change in age would change 0.863 units in respondents' RE. Other than those variables described above, could not found significantly contributing ORSs and hence, were not considered for step-wise multiple regressions.

\section{Step-wise multiple regression of independent variables with burnout}

Among burnout sub-scales, no variable was found significantly contributing to EX and CY. Therefore, only PE was considered for step-wise multiple regressions. The data on this aspect are presented in table 6.

\begin{tabular}{|l|l|l|l|l|l|}
\hline Sr. No. & Demographic variable & $\begin{array}{l}\text { Partial regression co-efficient } \\
\text { (bi) }\end{array}$ & S.E. of bi & 't' value & Significance value \\
\hline 1. & Level of Education & 1.674 & 0.827 & 2.024 & 0.044 \\
\hline
\end{tabular}

Table 6: Step-wise multiple regression of dependent variable with PE. ${ }^{2}=0.011$.

Only level of education was found significantly contributing to PE and hence, it was considered for step-wise multiple regressions. It is clear from the table that only level of education has explained $11 \%$ of total variation in respondents' $P E$. The ' $t$ ' value $(-2.024)$ was found significant at 0.05 level of significance indicating its significant contribution in PE. The partial regression co-efficient indicated that one unit change in age would change 0.827 unit in respondents' PE.

\section{Path analysis of demographic variables and organizational roles stress factors}

IRD: The data presented in Table 7 reveal that the variable age had exerted the highest positive direct effect on IRD followed by monthly income, experience, level of education and position. Age also exerted highly indirect effect on IRD followed by experience, level of education, position and monthly income. The variable viz., age was seen to have the highest positive first substantial effect on IRD through experience. The other important variables exerting substantial positive indirect effect upon IRD in descending orders were; experience through age, monthly income through age, level of education through age and position through monthly income. It can be thus, concluded that age was the important variable positively affecting IRD. Further, three variables viz., experience, level of education and monthly income exerted their substantial indirect effect all through age. Thus, age was emerged as an important variable.
RS: The data presented in Table 7 reveal that the variable age had exerted the highest positive direct effect on RS as path efficient followed by level of education, position, monthly income and experience. Monthly income exerted the highest positive indirect effect on RS followed by level of education, position, age and experience. The variable viz., age was seen to have the highest positive first substantial effect on RS through monthly income.

The other importance variables exerting substantial positive indirect effect upon RS in descending orders were; experience through age, level of education through position through level of education and monthly income through age. It can be thus, concluded that age was the important variable positively affecting RS. Further, two variables viz., experience and monthly income exerted their substantial indirect effect through age. Thus, age was emerged as an important variable.

REC: The data presented in Table 7 reveal that the variable, experience exerted the highest positive direct effect on REC as path efficient followed by position, monthly income, age and level of education. Experience also exerted highly indirect effect on REC followed by age, monthly income, level of education and position. The variable viz., experience was seen to have the highest positive first substantial effect on REC through age. The other importance variables exerting substantial positive indirect effect upon REC in descending orders were; age through experience, position through monthly income, monthly income through experience and level of education through position. It can be thus, concluded that experience was the 
Citation: Soni SR, Vyass JM, Pestonjee DM, Kher HN, Thakkar KA, et al. (2015) Effect of Demographic Variables on Organizational

Page 9 of 12

important variable positively affecting REC. Further, two variables viz., through experience. Thus, experience was emerged as an important age and monthly income exerted their substantial indirect effect variable.

\begin{tabular}{|c|c|c|c|c|c|}
\hline \multirow{2}{*}{$\begin{array}{l}\text { ORS } \\
(n=382)\end{array}$} & \multicolumn{5}{|c|}{ Demographic variables } \\
\hline & Position (X1) & $\begin{array}{l}\text { Experience } \\
(\mathrm{X} 2)\end{array}$ & $\begin{array}{l}\text { Age } \\
(\mathrm{X} 3)\end{array}$ & $\begin{array}{l}\text { Level of education } \\
\text { (X4) }\end{array}$ & $\begin{array}{l}\text { Monthly income } \\
\text { (X5) }\end{array}$ \\
\hline \multirow{3}{*}{ IRD } & 0.0261 & 0.1135 & 0.2440 & 0.0546 & 0.1242 \\
\hline & 0.0222 & 0.1895 & 0.2434 & 0.0505 & 0.0120 \\
\hline & $0.0325(X 5)$ & $0.2125(X 3)$ & $0.2440(X 2)$ & $0.0546(X 3)$ & $0.1242(X 3)$ \\
\hline \multirow{3}{*}{ RS } & 0.1167 & 0.0055 & 0.1692 & 0.1206 & 0.1054 \\
\hline & 0.0667 & 0.0344 & 0.0462 & 0.0826 & 0.1024 \\
\hline & $0.1167(X 4)$ & $0.1474(X 3)$ & 0.1692 (X5) & $0.1206(X 1)$ & $0.1054(X 3)$ \\
\hline \multirow{3}{*}{ REC } & 0.0451 & 0.0652 & 0.0288 & 0.0018 & 0.0289 \\
\hline & 0.0081 & 0.0418 & 0.0402 & 0.0145 & 0.0338 \\
\hline & $0.0451(X 5)$ & $0.0652(X 3)$ & $0.0568(X 2)$ & $0.0205(X 1)$ & $0.0342(X 2)$ \\
\hline \multirow{3}{*}{ RE } & 0.0014 & 0.0070 & 0.0281 & 0.1376 & 0.0201 \\
\hline & 0.095 & 0.0329 & 0.0041 & 0.0024 & 0.0259 \\
\hline & $0.0625(X 5)$ & $0.0359(X 4)$ & $0.0281(X 5)$ & $0.1376(X 3)$ & $0.0201(X 3)$ \\
\hline \multirow{3}{*}{$\mathrm{RI}$} & 0.0221 & 0.1025 & 0.0207 & 0.0328 & 0.0633 \\
\hline & 0.0101 & 0.0075 & 0.0113 & 0.0242 & 0.0584 \\
\hline & $0.0221(X 5)$ & $0.1025(X 5)$ & $0.0893(X 2)$ & $0.0328(X 2)$ & $0.0633(X 2)$ \\
\hline \multirow{3}{*}{$\mathrm{Pl}$} & 0.0205 & 0.2349 & 0.3680 & 0.0479 & 0.0320 \\
\hline & 0.0175 & 0.2065 & 0.181 & 0.0613 & 0.0902 \\
\hline & $0.0335(X 3)$ & $0.3205(X 3)$ & $0.3680(X 2)$ & $0.0613(X 2)$ & $0.2112(X 3)$ \\
\hline \multirow{3}{*}{ SRD } & 0.0691 & 0.0258 & 0.0999 & 0.0620 & 0.0540 \\
\hline & 0.0321 & 0.0410 & 0.0429 & 0.0260 & 0.0540 \\
\hline & $0.0691(X 4)$ & $0.0870(X 3)$ & 0.0999 (X5) & $0.0620(X 1)$ & $0.0573(X 3)$ \\
\hline \multirow{3}{*}{ RA } & 0.0829 & 0.1774 & 0.2117 & 0.0570 & 0.0301 \\
\hline & 0.0091 & 0.1434 & 0.1257 & 0.0420 & 0.0465 \\
\hline & $0.0829(X 4)$ & $0.1774(X 3)$ & $0.2117(\mathrm{X} 2)$ & $0.0570(X 2)$ & $0.1215(X 3)$ \\
\hline \multirow{3}{*}{ RIN } & 0.0108 & 0.0109 & 0.2296 & 0.0491 & 0.1789 \\
\hline & 0.0329 & 0.0118 & 0.1166 & 0.0351 & 0.1279 \\
\hline & $0.0469(X 5)$ & $0.2000(X 3)$ & 0.2296 (X5) & $0.0491(X 5)$ & $0.1789(X 3)$ \\
\hline \multirow{3}{*}{ RO } & 0.0340 & 0.1717 & 0.0631 & 0.0057 & 0.1044 \\
\hline & 0.037 & 0.1097 & 0.1265 & 0.0152 & 0.0454 \\
\hline & $0.0340(X 5)$ & $0.1717(X 3)$ & $0.1495(X 2)$ & $0.0448(X 2)$ & $0.1044(X 2)$ \\
\hline
\end{tabular}

Table 7: Path analysis of demographic variables on ORS factors, $D=$ Direct effect, $T=$ Total indirect effect, $S=$ Substantial indirect effect

RE: The data presented in Table 7 reveal that the variable, level of efficient followed by age, monthly income, experience and position. education exerted the highest positive direct effect on RE as path Position exerted the highest positive indirect effect on RE followed by 
experience, monthly income, age and level of education. The variable viz., level of education was seen to have the highest positive first substantial effect on RE through age. The other important variables exerting substantial positive indirect effect upon RE in descending orders were; position through monthly income, experience through level of education, age through monthly income and monthly income through age. It can be thus, concluded that level of education was the important variable positively affecting RE. Further, two variables viz., age and position exerted their substantial indirect effect through monthly income, and level of education and monthly income exerted their substantial indirect effect through age. Thus, level of education was emerged as an important variable.

RI: The data presented in Table 7 reveal that the variable experience had exerted the highest positive direct effect on RI as path efficient followed by monthly income, level of education, position and age. Monthly income also exerted highly indirect effect on RI followed by level of education, age, position and experience. The variable viz., experience was seen to have the highest positive first substantial effect on RI through monthly income. The other important variables exerting substantial positive indirect effect upon RI in descending orders were; age through experience, monthly income through experience, level of education through experience and position through monthly income. It can be thus concluded that experience was the important variable positively affecting RI. Further, three variables viz., age, level of education and monthly income exerted their substantial indirect effect all through experience. Thus, experience was emerged as an important variable.

PI: The data presented in Table 7 reveal that the variable age had exerted the highest positive direct effect on PI as path efficient followed by experience, level of education, monthly income and position. Experience also exerted highly indirect effect on PI followed by age, monthly income, level of education and position. The variable viz., age was seen to have the highest positive first substantial effect on PI through experience. The other important variables exerting substantial positive indirect effect upon PI in descending orders were; experience through age, monthly income through age, level of education through experience and position through age. It can be thus concluded that age was the important variable positively affecting PI. Further, three variables viz., position, experience and monthly income exerted their substantial indirect effect all through age. Thus, age was emerged as an important variable.

SRD: The data presented in Table 7 reveal that the variable age had exerted the highest positive direct effect on SRD as path efficient followed by position, level of education, monthly income and experience. Monthly income also exerted highly indirect effect on SRD followed by age, experience, position and level of education. The variable viz., age was seen to have the highest positive first substantial effect on SRD through monthly income. The other important variables exerting substantial positive indirect effect upon SRD in descending orders were; experience through age, position through level of education, level of education through position and monthly income through age. It can be thus concluded that age was the important variable positively affecting SRD. Further, two variables viz., experience and monthly income exerted their substantial indirect effect through age. Thus, age was emerged as an important variable.

RA: The data presented in Table 7 reveal that the variable age had exerted the highest positive direct effect on RA as path efficient followed by experience, position, level of education and monthly income. Experience also exerted highly indirect effect on RA followed by age, monthly income, level of education and position. The variable viz., age was seen to have the highest positive first substantial effect on RA through experience. The other important variables exerting substantial positive indirect effect upon RA in descending orders were; experience through age, monthly income through age, position through level of education and level of education through experience. It can be, thus, concluded that age was the important variable positively affecting RA. Further, two variables viz., experience and monthly income exerted their substantial indirect effect through age. Thus, age was emerged as an important variable.

RIN: The data presented in Table 7 reveal that the variable age had exerted the highest positive direct effect on RIN as path efficient followed by monthly income, level of education, experience and position. Monthly income also exerted highly indirect effect on RIN followed by age, level of education, position and experience. The variable viz., age was seen to have the highest positive first substantial effect on RIN through monthly income. The other important variables exerting substantial positive indirect effect upon RIN in descending orders were; experience through age, monthly income through age, level of education through monthly income and position through monthly income. It can be, thus, concluded that age was the important variable positively affecting RIN. Further, two variables viz., experience and monthly income exerted their substantial indirect effect through age. Thus, age was emerged as an important variable.

RO: The data presented in Table 7 reveal that the variable experience had exerted the highest positive direct effect on RO as path efficient followed by monthly income, age, position and level of education. Age also exerted highly indirect effect on RO followed by experience, monthly income, level of education and position. The variable viz., experience was seen to have the highest positive first substantial effect on RO through age. The other important variables exerting substantial positive indirect effect upon $\mathrm{RO}$ in descending orders were; age through experience, monthly income through experience, level of education through experience and position through monthly income. It can be, thus, concluded that experience was the important variable positively affecting RO. Further, three variables viz., age, monthly income and level of education exerted their substantial indirect effect all through experience. Thus, experience was emerged as an important variable.

\section{Path analysis of demographic variables and burnout}

EX: The data presented in Table 8 and figure 4 reveal that the variable position had exerted the highest positive direct effect on EX as path efficient followed by experience, level of education, age and monthly income. Position also exerted highly indirect effect on EX followed by level of education, age, experience and monthly income. The variable viz., position was seen to have the highest positive first substantial effect on EX through level of education. The other important variables exerting substantial positive indirect effect upon EX in descending orders were, experience through age, age through experience, level of education through position and monthly income through experience. It can be, thus, concluded that position was the important variable positively affecting EX. Further, the variables viz., level of education exerted its substantial indirect effect through position. Thus, position was emerged as an important variable. 


\begin{tabular}{|c|c|c|c|c|c|}
\hline \multirow[b]{2}{*}{ Burnout } & \multicolumn{5}{|c|}{ Demographic variables } \\
\hline & Position (X1) & $\begin{array}{l}\text { Experience } \\
\text { (X2) }\end{array}$ & $\begin{array}{l}\text { Age } \\
(X 3)\end{array}$ & $\begin{array}{l}\text { Level of education } \\
\text { (X4) }\end{array}$ & $\begin{array}{l}\text { Monthly income } \\
\text { (X5) }\end{array}$ \\
\hline \multirow{3}{*}{ EX } & 0.1124 & 0.0698 & 0.0388 & 0.0603 & 0.0131 \\
\hline & 0.0374 & 0.0298 & 0.0318 & 0.0343 & 0.0116 \\
\hline & $0.1124 \times 4)$ & $0.0698(X 3)$ & $0.0608(X 2)$ & $0.0603(X 1)$ & $0.0366(X 2)$ \\
\hline \multirow{3}{*}{$\mathrm{CY}$} & 0.0259 & 0.0409 & 0.0147 & 0.1108 & 0.0456 \\
\hline & 0.0383 & 0.0390 & 0.0216 & 0.0778 & 0.0336 \\
\hline & $0.0503 \times 4)$ & 0.0409 (X4) & $0.0356(X 2)$ & $0.1108(X 1)$ & $0.0456(X 2)$ \\
\hline \multirow{3}{*}{ PE } & 0.0410 & 0.1603 & 0.1709 & 0.1416 & 0.1044 \\
\hline & 0.0413 & 0.1293 & 0.1559 & 0.0386 & 0.0114 \\
\hline & $0.0643 \times 4)$ & $0.1603(X 3)$ & $0.1709(X 2)$ & $0.1416(X 2)$ & $0.1044(X 3)$ \\
\hline
\end{tabular}

Table 8: Path analysis of demographic variables on Burnout, $D=$ Direct effect, $T=$ Total indirect effect, $S=$ Substantial indirect effect

CY: The data presented in Table 8 reveal that the variable level of education had exerted the highest positive direct effect on CY as path efficient followed by monthly income, experience, position and age. Level of education also exerted highly indirect effect on CY followed by experience, position, monthly income and age. The variable viz., level of education was seen to have the highest positive first substantial effect on CY through position. The other important variables exerting substantial positive indirect effect upon CY in descending orders were; position through level of education, monthly income through experience, experience through level of education and age through experience. It can be, thus, concluded that level of education was the important variable positively affecting CY. Further, two variables viz., position and experience exerted their substantial indirect effect through level of education. Thus, level of education was emerged as an important variable.

PE: The data presented in Table 8 reveal that the variable age had exerted the highest positive direct effect on PE as path efficient followed by experience, level of education, monthly income and position. Age also exerted highly indirect effect on PE followed by experience, position, level of education and monthly income. The variable viz., age was seen to have the highest positive first substantial indirect effect on PE through experience. The other important variables exerting substantial positive indirect effect upon PE in descending orders were; experience through age, level of education through experience, monthly income through age and position through level of education. It can be, thus, concluded that age was the important variable positively affecting PE. Further, two variables viz., experience and monthly income exerted their substantial indirect effect through age. Thus, age was emerged as an important variable.

\section{Conclusion}

On the basis of forgoing discussion, majority of veterinarians and para veterinarians fall under "high" category when it comes to role stress like IRD, RS, REC, RO, PI and RA. This shows that majority of the employees are under stress due to conflict that is arising between their professional life and non-work life. They also feel that their abilities are being misused or under used in their job. They become restless because of the conflicting expectations of their seniors, juniors and peers. This also shows that the demands on majority of the employees are much more than what they can tolerate. A high score on PI and RIN shows that majority of the employees feel that they do not have sufficient skills and sufficient/adequate resources to perform their duties effectively. This shows that there is high priority to boost the motivation of the employees by providing them with sufficient training and resources. The RS score in the scale shows that there is an immediate need to provide ample opportunities for the employee's growth and learning. Once this is done the stress arising among the employees due to other factors can reduce tremendously. A low score on RE shows that the employees have good working environment where there is no conflict among the employees due to the nature of work. The overall results in the ORS scale indicate that employees are having high role stress and need immediate interventions to mitigate the adverse effect of the role stress.

In case of burnout, existence of majority of employees in the low category for the "exhaustion" factor indicates that they are experiencing a low fatigue which is a healthy sign. In terms of their attitude to work, majority of the employees are in "medium" category which is a cautioning sign. If measures are taken properly there is a scope to turn their energies positively towards their profession, otherwise, they can even turn completely indifferent towards their work. Looking to professional Efficacy, majority of employees are in "high" category of professional efficacy which shows that the employees stress levels have not reached the peak level. The results indicate that veterinarians and paraveterinarians are having moderate level of cynicism and need certain interventions to mitigate the possibilities of adverse effect of the burnout.

Length of service has negatively significant relationship with RS, PI and REC and age had negatively significant relationship with RS, PI and RI. Educational qualification had positively significant relationship only with $\mathrm{RE}$ and monthly income had negatively significant relationship only with PI. All the six demographic variables viz., position, experience, age, marital status, educational qualification and monthly income failed to establish significant relationship with three sub-scales of burnout viz., EX, CY and PE. All the ten ORSs 
established positively significant relationship with both EX and CY. However, they failed to establish significant relationship with PE. Age exerted the highest direct effect upon IRD, RS, PI, SRD, RA and RIN, whereas experience exerted the highest direct effect upon REC and RI. Educational qualification exerted highest direct effect upon RE. Position and educational qualification exerted highest direct effect upon EX and CY, respectively, whereas age exerted highest direct effect upon PE.

The overall analysis reveals that there are ten underlying role related factors which represent the different variables considered originally in the present study. The theoretical significance of the findings of this study is noteworthy as it has tried to explore the antecedents of organizational role stress in the animal husbandry. It has also pointed out to give emphasis on providing opportunities for learning, training and development to its employees. Tenure (length) of service plays a vital role in the reduction of role stress in an organization. Stress beyond a certain level always poses threat to the quality of work life as well as physical and psychological well-being. A high level of occupational stress, not only detrimentally influence the quality, productivity and creativity of the employees but also employee's health, well being and morale. Hence, job related stress needs to be identified and addressed as early as possible. The results lead us to conclude that there is a need for more proactive HR policies to maintain and alleviate the role stress and burnout. It can be recommended that the organization needs to mandate a 'stress audit' for various categories of personnel. Counseling may also be provided on a case to case basis so that individuals may be helped with their cognitive restructuring.

\section{References}

1. Agrawal UN., Malhan NK, Singh B (1979) Some classifications of stress and its applications at work. Ind. J. Indus. Rel 15: 41-50.

2. Pestonjee DM (1992) Stress and Coping: The Indian Experience. 2nd edition, Sage Publication, New Delhi.

3. Khanna B (1985) Relationship between organizational climate and organizational role stress and their impact upon organizational effectiveness: A case study. Banaras Hindu University, Varanasi, India.

4. McCarthy P (1985) Burnout in psychiatric nursing. J Adv Nursing 10: 305-310.
5. Price L, Spence SH (1994) Burnout symptoms amongst drug and alcohol service employees: Gender differences in the interaction between work and home stressors. Anxiety, Stress and Coping. 7: 67-84.

6. Mishra P, Rani D (2001) Occupational stress among working women in emerging services. Management and Labour Studies 26: 25-36.

7. Hassanzadeh R, Khalilian A, Enayati T (2004) The relationship between $\mathrm{A}$ and $\mathrm{B}$ personality types and job burnout among principals. The $\mathrm{J}$ Indian Acad. Appl. Psychol 3: 51-57.

8. Ansari MR (1991) An investigation into the stress of agriculture university teachers. Ph.D. Thesis, I.A.R.I., New Delhi, India.

9. Ansari MR, Singh RP (1997) A study on nature and extent of stress in teachers and impact of moderators on stress. J Extn Edn 8: 1623-1625.

10. Bhagawan S (1997) Job stress and burn out in teachers of secondary school in Orissa. J. Educational. Res. Extn 33: 218-234.

11. Bhatia P, Kumar A (2005) Occupational stress and burn out in industrial employees. Indian Psy. Rev 64: 191-198.

12. Chand P, Monga OP (2007) Correlates of job stress and burn out. J.Com Gui. Res 24: 243-252.

13. Upadhyay BK, Singh B (1999) Experience of stress: Differences between college teachers and executives. Psy Stu 44: 65-68.

14. Kumar P, Bohra C (1979) Job satisfaction and perceived organizational climate. Indian J. Social Work 40: 23-26.

15. Pareek U (1993) Role stress and coping: A framework In: Studies in Organizational Role Stress and Coping edited by Pareek U and Pestonjee DM, Rawat Publications, Jaipur: 1-40.

16. Maslach C, Jackson S, Leiter MP (1996) Maslach Burnout Inventory Manual, 3rd Ed., CPP, Inc., Mountain view, California

17. Dewey DR, Lu KH (1959) A correlation and path coefficient analysis of components of crested wheatgrass seed production. Agron. J 51: 515-518.

18. Efroymson MA (1960) "Multiple Regression Analysis." In Ralston, A. and Wilf, H.S., Editors, Mathematical Methods for Digital Computers. Wiley.

19. Maslach C (2003) Burnout: the cost of caring, Malor Books, ISBN $=9781883536350$

20. Maslach C, Jackson S (1986) Maslach Burnout Inventory, 2nd Ed. Consulting Psychologist Press, Palo Alto, CA, U.S.A.

21. Pareek U (1993) Making Organizational Roles Effective. TATA McGrawHill Publ. Co. Ltd., New Delhi. pp. 68-70.

22. Twelfth five year plan (2012-17) of Animal husbandry department, Gujarat Government. 